Supporting Information:

\title{
Rapid Prediction of Bimetallic Mixing Behavior at the Nanoscale
}

James Dean+, Michael J. Cowan+, Jonathan Estes, Mahmoud Ramadan and Giannis Mpourmpakis*

Department of Chemical Engineering, University of Pittsburgh, Pittsburgh, PA 15261, USA

+Equal contribution

*Corresponding author email: gmpourmp@pitt.edu 


\section{Section 1. Connecting Excess Energy with Enthalpy of Mixing}

In their development of the BC model, Yan et al ${ }^{1}$ showed that for a bimetallic Nanoparticle (NP), the Excess Energy (EE) of an arbitrary alloy is related to the cohesive energy of that alloy and its monometallic counterparts, scaled by the fraction $\left(F_{A}\right.$ and $\left.F_{B}\right)$ of that component in the NP (Equation 1).

$$
E E_{A_{x} B_{y}}=C E_{A_{x} B_{y}}-\left(F_{A} C E_{A_{x+y}}+F_{B} C E_{B_{x+y}}\right)
$$

Recalling the definition of enthalpy (Equation2), $\mathrm{U}$ is related to $\mathrm{H}$ via the product of pressure $(\mathrm{P})$ and volume (V). When pressure is $\mathrm{O}, \mathrm{H}$ and $\mathrm{U}$ are therefore equivalent.

$$
H=U+P V
$$

The enthalpy of mixing for a binary system is defined as the difference between the enthalpy of the mixed system $\left(\mathrm{H}_{\mathrm{ij}}\right)$ and the product of the enthalpy of the pure systems $\left(\mathrm{H}_{\mathrm{i}}\right.$ and $\left.\mathrm{H}_{\mathrm{j}}\right)$ with their fractions $\left(\mathrm{F}_{\mathrm{i}}\right.$ and $\left.\mathrm{F}_{\mathrm{j}}\right)$ in the mixed system (Equation 3).

$$
\Delta H_{\text {mix }}=H_{i j}-\left(F_{i} H_{i}+F_{j} H_{j}\right)
$$

At 0 pressure, $\mathrm{H}=\mathrm{U}$ as a consequence of Equation 2. This allows us to find Equation 4.

$$
\Delta H_{\text {mix }}=U_{A B}-\left(F_{A} U_{B}+F_{A} U_{B}\right)
$$

Under the framework of the $\mathrm{BC}$ model, the energy holding a NP together is exclusively defined as the sum of the bonds in the NP. We can use this to approximate the internal energy of the system as the product of the number of atoms in the system $(\mathrm{N})$ and the cohesive energy (Equation 5 ). This comes from the definition of $\mathrm{CE}$ as the average energy (on a per-atom basis) holding the system together.

$$
N * C E_{A_{x} B_{y}} \approx U_{A_{x} B_{y}}
$$

We can then plug the approximation found in Equation 5 into Equation 1 to generate Equation 6.

$$
E E_{\text {alloy }} \approx\left(\frac{U_{A_{x} B_{y}}}{N}-\left(\frac{F_{A} U_{A_{N}}}{N}+\frac{F_{B} U_{B_{N}}}{N}\right)\right)=\frac{U_{A_{x} B_{y}}-\left(F_{A} U_{A_{N}}+F_{B} U_{B_{N}}\right)}{N}
$$

Recalling the relationship between $\Delta H_{m i x}$ and $U$ derived in Equation 4, we can further simplify Equation 6 , yielding a relationship between the EE and enthalpy of mixing (Equation 7).

$$
E E_{\text {alloy }} \approx \frac{\Delta H_{\text {mix }}}{N}
$$

Overall, this allows us to relate the property calculated by the BC Model (CE) with the important thermodynamic property $\Delta H_{\text {mix }}$, which allows us to then approximate the free energy of mixing in the main document. 
Section 2. Statistical Thermodynamics

The Zero-Point Energy (ZPE) was calculated via Equation $8,{ }^{2}$ where $h$ represents Planck's constant and $v$ represents the vibrational frequency. It is a summation over the $\mathrm{N}_{\text {modes }}$ vibrational modes.

$$
Z P E=\sum_{i=1}^{N_{\text {modes }}} \frac{h v_{i}}{2}
$$

The constant-volume heat capacity due to vibrational motion $\left(\mathrm{C}_{v, \text { vib }}\right)$ was calculated via Equation 9 , where T refers to temperature and $k_{b}$ refers to Boltzmann's constant. Utilizing the energy of each vibrational mode ${ }^{3}$ we sum over all modes to find the vibrational heat capacity.

$$
\int_{0}^{T} C_{V, v i b} d T=\sum_{i=1}^{N_{\text {modes }}} \frac{h v_{i}}{e^{\frac{h v_{i}}{k_{b} T}}-1}
$$

The entropic contribution from entropy $\left(S_{v}\right)$ was calculated via Equation $10{ }^{2}$

$$
S_{V}=\sum_{i=1}^{N_{\text {modes }}} \frac{h v_{i}}{k_{b} T\left(e^{\frac{h v_{i}}{k_{b} T}}-1\right)}-\ln \left[1-e^{-\frac{h v_{i}}{k_{b} T}}\right]
$$

Finally, Gibb's free energy (G) can be calculated via Equation 11.

$$
G=E_{D F T}+Z P E+C_{V} T-T S_{V}
$$

\begin{tabular}{|c|c|c|c|c|c|c|}
\hline System & Sample & DFT (eV) & ZPE (eV) & $\mathrm{C}_{\mathrm{V}, \mathrm{vib}} \mathrm{T}(\mathrm{eV}), 298 \mathrm{~K}$ & TS $(\mathrm{eV}), 298 \mathrm{~K}$ & G (eV), 298K \\
\hline $\mathrm{Ag}_{50} \mathrm{Au}_{5}$ & 1 & -54879.961 & 0.914 & 3.249 & 7.769 & -54883.567 \\
\hline $\mathrm{Ag}_{50} \mathrm{Au}_{5}$ & 2 & -54879.476 & 0.905 & 3.256 & 7.808 & -54883.123 \\
\hline $\mathrm{Ag}_{50} \mathrm{Au}_{5}$ & 3 & -54879.984 & 0.915 & 3.248 & 7.765 & -54883.586 \\
\hline $\mathrm{Ag}_{25} \mathrm{Au}_{30}$ & 1 & -52310.650 & 0.836 & 3.316 & 8.226 & -52314.724 \\
\hline $\mathrm{Ag}_{25} \mathrm{Au}_{30}$ & 2 & -52310.755 & 0.828 & 3.323 & 8.286 & -52314.890 \\
\hline $\mathrm{Ag}_{25} \mathrm{Au}_{30}$ & 3 & -52311.050 & 0.838 & 3.315 & 8.232 & -52315.129 \\
\hline $\mathrm{Ag}_{20} \mathrm{Au}_{35}$ & 1 & -51796.847 & 0.827 & 3.324 & 8.295 & -51800.991 \\
\hline $\mathrm{Ag}_{20} \mathrm{Au}_{35}$ & 2 & -51796.329 & 0.819 & 3.331 & 8.344 & -51800.522 \\
\hline $\mathrm{Ag}_{20} \mathrm{Au}_{35}$ & 3 & -51796.790 & 0.830 & 3.322 & 8.286 & -51800.924 \\
\hline $\mathrm{Ag}_{5} \mathrm{Au}_{50}$ & 1 & -50250.900 & 0.760 & 3.381 & 8.676 & -50255.435 \\
\hline $\mathrm{Ag}_{5} \mathrm{Au}_{50}$ & 2 & -50251.452 & 0.769 & 3.373 & 8.618 & -50255.927 \\
\hline $\mathrm{Ag}_{5} \mathrm{Au}_{50}$ & 3 & -50251.458 & 0.774 & 3.370 & 8.727 & -50256.040 \\
\hline
\end{tabular}

Table S1. Thermodynamic data for several chemical orderings randomly sampled in various $\mathrm{Ag}_{\mathrm{x}} \mathrm{Au} \mathrm{u}_{55-\mathrm{x}}$ systems. 
Table S2. Thermodynamic data for several chemical orderings randomly sampled in various $\mathrm{Ag}_{\mathrm{x}} A \mathrm{u}_{13-\mathrm{x}}$ systems.

\begin{tabular}{lcccccc} 
System & Sample & DFT (eV) & ZPE $(e V)$ & $C_{V, v i b}(e V), 298 K$ & $T S(e V), 298 K$ & $\mathbf{G}(e V), 298 K$ \\
\hline $\mathrm{Ag}_{11} \mathrm{Au}_{2}$ & 1 & -12882.312 & 0.185 & 0.679 & 1.663 & -12883.111 \\
$\mathrm{Ag}_{11} \mathrm{Au}_{2}$ & 2 & -12881.719 & 0.180 & 0.682 & 1.671 & -12882.528 \\
$\mathrm{Ag}_{6} \mathrm{Au}_{7}$ & 1 & -12368.686 & 0.174 & 0.689 & 1.761 & -12369.584 \\
$\mathrm{Ag}_{6} \mathrm{Au}_{7}$ & 2 & -12368.626 & 0.177 & 0.686 & 1.735 & -12369.498 \\
$\mathrm{Ag}_{6} \mathrm{Au}_{7}$ & 3 & -12368.250 & 0.174 & 0.688 & 1.718 & -12369.106 \\
$\mathrm{Ag}_{6} \mathrm{Au}_{7}$ & 4 & -12369.351 & 0.182 & 0.682 & 1.686 & -12370.174 \\
$\mathrm{Ag}_{2} \mathrm{Au}_{11}$ & 1 & -11955.575 & 0.156 & 0.703 & 1.783 & -11956.499 \\
$\mathrm{Ag}_{2} \mathrm{Au}_{11}$ & 2 & -11955.575 & 0.156 & 0.703 & 1.786 & -11956.501 \\
$\mathrm{Ag}_{2} \mathrm{Au}_{11}$ & 3 & -11955.915 & 0.161 & 0.700 & 1.840 & -11956.894
\end{tabular}




\section{Section 3. Comparing NP Mixing Behavior to Fully Random Mixing}

For bimetallic systems, we define fully random mixing as the equal likelihood of forming any bond type (i.e. no energetic preference between bond types). To determine the bond fractions $\left(F_{A-A}, F_{B-B}\right.$, and $\left.F_{A-B}\right)$ of a bimetallic NP that exhibits fully random mixing, we can calculate the probability of consecutively selecting two atoms from the group in order to form a bond. First, we imagine a system of $\mathrm{N}_{\text {Atoms }}$ atoms consisting of $A$ and $B$ atom types, where the composition is defined by $x_{A}+x_{B}=1$. The probability of creating an $A-A$ bond $\left(p_{A-A}\right)$ is the chance of randomly choosing an $A$ atom followed by choosing a second $A$ atom, as shown in Equation 12. The same reasoning can be used to determine the fraction of $B$-B bonds (Equation 13).

$$
\begin{aligned}
& F_{A-A}=p_{A-A}=x_{A} \times\left(\frac{N_{A}-1}{N_{\text {Atoms }}-1}\right)=x_{A} \times\left(\frac{N_{A}}{N_{\text {Atoms }}-1}-\frac{1}{N_{\text {Atoms }}-1}\right) \\
& F_{B-B}=p_{B-B}=x_{B} \times\left(\frac{N_{B}-1}{N_{\text {Atoms }}-1}\right)=x_{B} \times\left(\frac{N_{B}}{N_{\text {Atoms }}-1}-\frac{1}{N_{\text {Atoms }}-1}\right)
\end{aligned}
$$

This same reasoning can be used to develop an expression of the total number of hetero-atomic bonds in the system (Equations 14-16).

$$
\begin{gathered}
p_{A-B}=x_{A}\left(\frac{N_{B}}{N_{\text {Atoms }}-1}\right) \\
p_{B-A}=x_{B}\left(\frac{N_{A}}{N_{\text {Atoms }}-1}\right) \\
F_{A-B}=F_{A-B \| B-A}=p_{A-B}+p_{B-A}=x_{A}\left(\frac{N_{B}}{N_{\text {Atoms }}-1}\right)+x_{B}\left(\frac{N_{A}}{N_{\text {Atoms }}-1}\right)
\end{gathered}
$$

For a sufficiently large NP (i.e. $\mathrm{N}_{\text {Atoms }} \gg 1$ ), we can simplify several terms in Equations 12-16 using Equations 17 and 18.

$$
\begin{gathered}
\lim _{N_{\text {Atoms }} \rightarrow \infty} \frac{N_{A}}{N_{\text {Atoms }}-1}=\frac{N_{A}}{N_{\text {Atoms }}}=x_{A} \\
\lim _{N_{\text {Atoms }} \rightarrow \infty} \frac{1}{N_{\text {Atoms }}-1}=0
\end{gathered}
$$

Thus, for sufficiently large NPs, we can simplify Equations 12, 13, and 16 to Equations 19-21.

$$
\begin{gathered}
F_{A-A}=x_{A}^{2} \\
F_{B-B}=x_{B}^{2} \\
F_{A-B}=x_{A} x_{B}+x_{B} x_{A}=2 x_{A} x_{B}
\end{gathered}
$$

This formulation is equivalent to applying a mean-field approximation with equal pairwise energies in a regular solution binary mixture. 


\section{Section 4. Boltzmann Statistics at Various Temperatures}

In Figure 6 of the main document, we present morphology phase diagrams at 298K for $\mathrm{AuCu}, \mathrm{AgAu}$, and AgCu systems with icosahedral, cuboctahedral, or EPB structures. In this section, we report the same type of diagram, at 77K (Figure S1), 640K (Figure S2), and 1073K (Figure S3). Overall, we observe that as temperature increases, the structural trend tends toward an even mix of all three morphologies. At low temperatures, the thermodynamically-most-favorable structures tend to dominate the distribution.

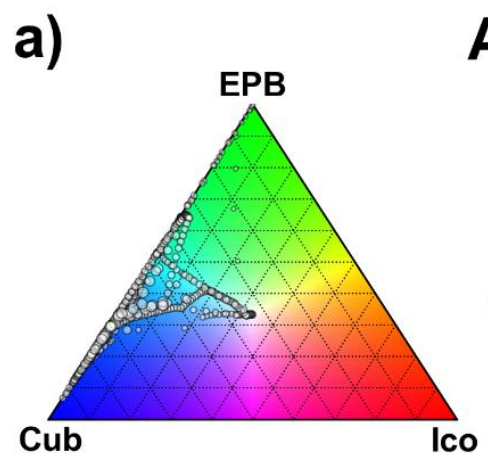

\section{$\mathrm{AuCu}, 77 \mathrm{~K}$}
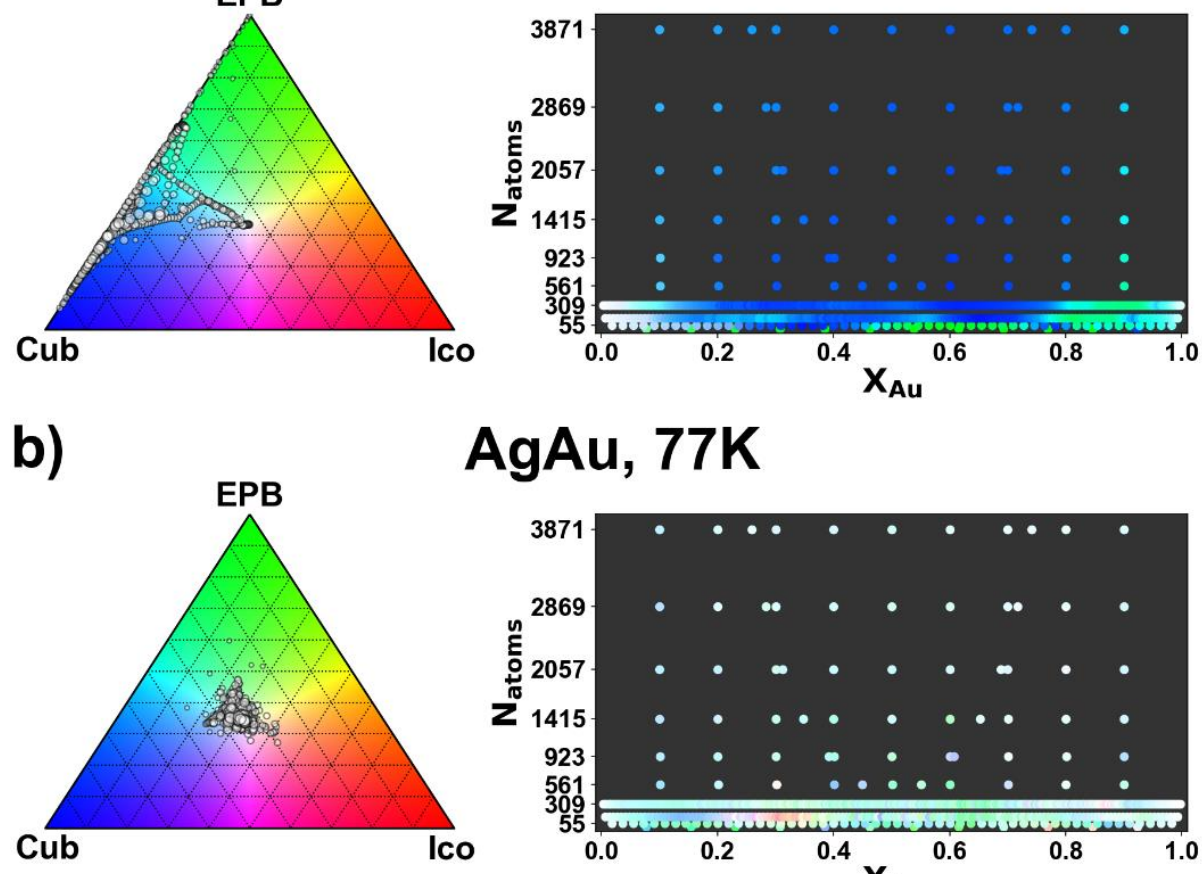

AgAu, 77K
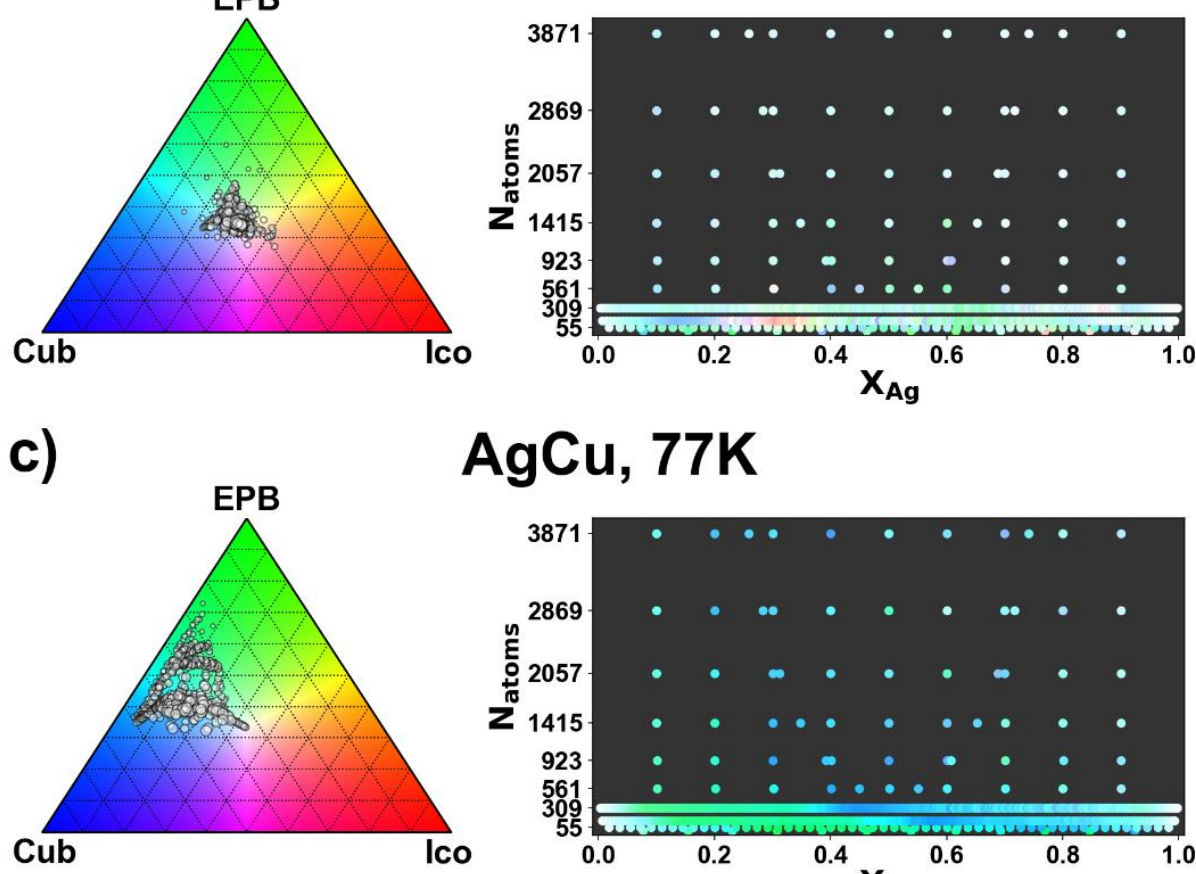

$\mathrm{AgCu}, 77 \mathrm{~K}$

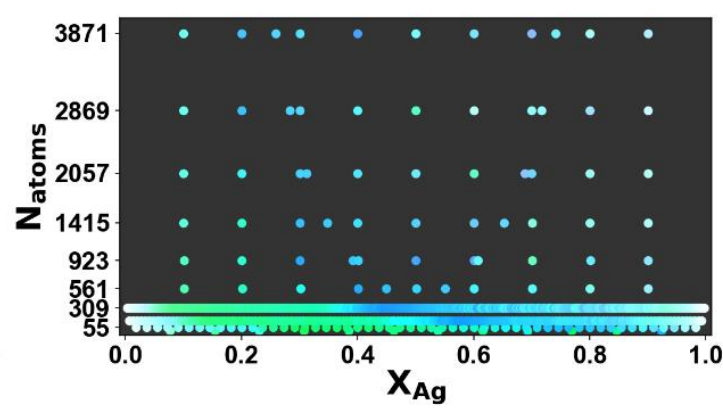

Figure S1. Morphology phase diagrams for the a) $\mathrm{AuCu}$, b) $\mathrm{AgAu}$, and c) AgCu systems at 77K. The righthand column plots the preferred morphological phase as a function of the number of atoms and composition of the system. The legend is given by the ternary diagrams on the left-hand side, which show the percentage of the Boltzmann population taking on cuboctahedral, icosahedral, or EPB. White points, for example, indicate all three morphologies are equally favorable. In addition, the size of the points on the ternary diagrams corresponds to NP size. 


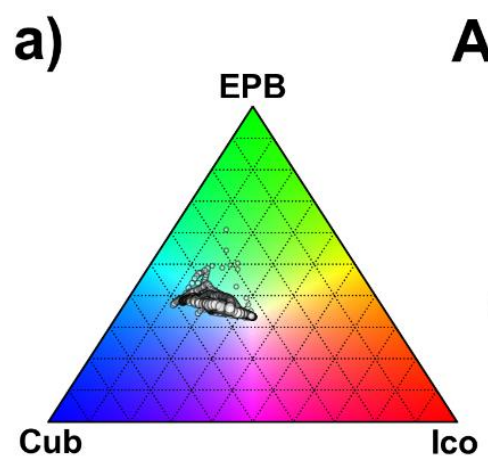

$\mathrm{AuCu}, 640 \mathrm{~K}$
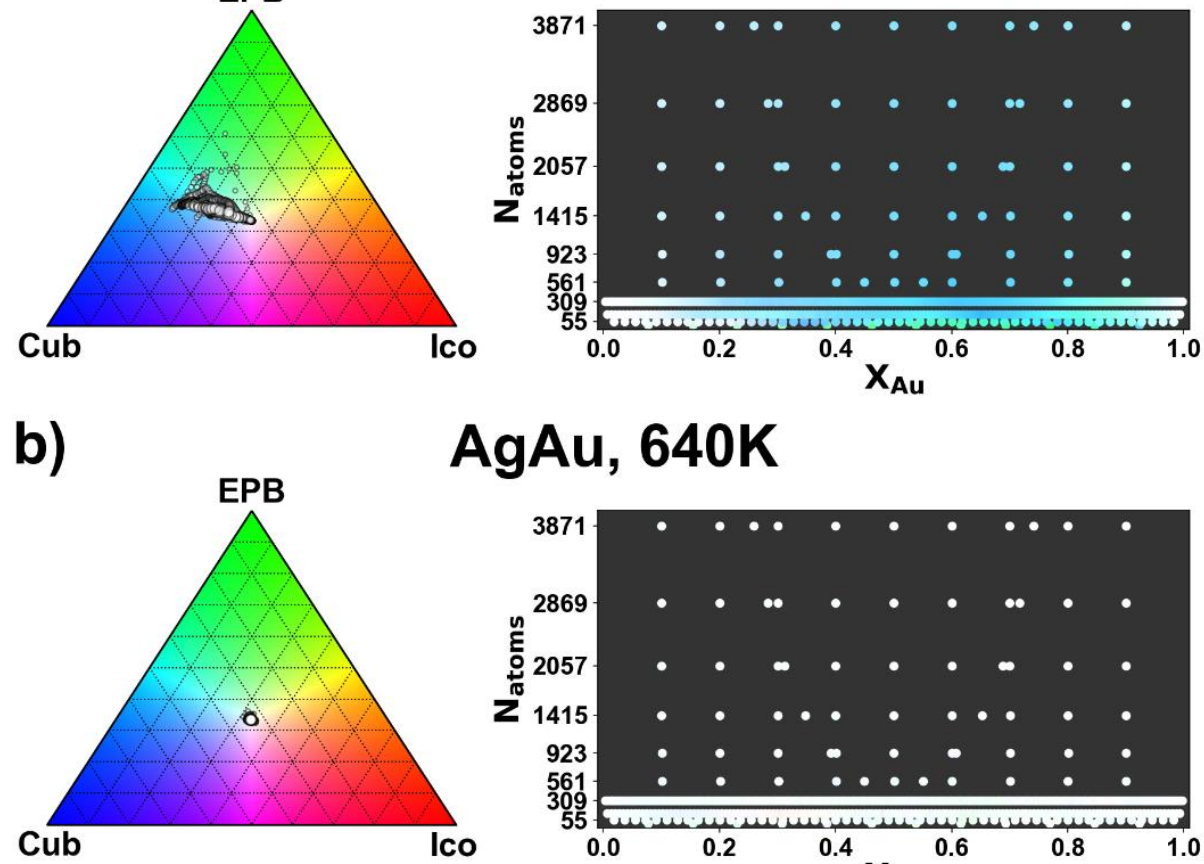

AgAu, 640K
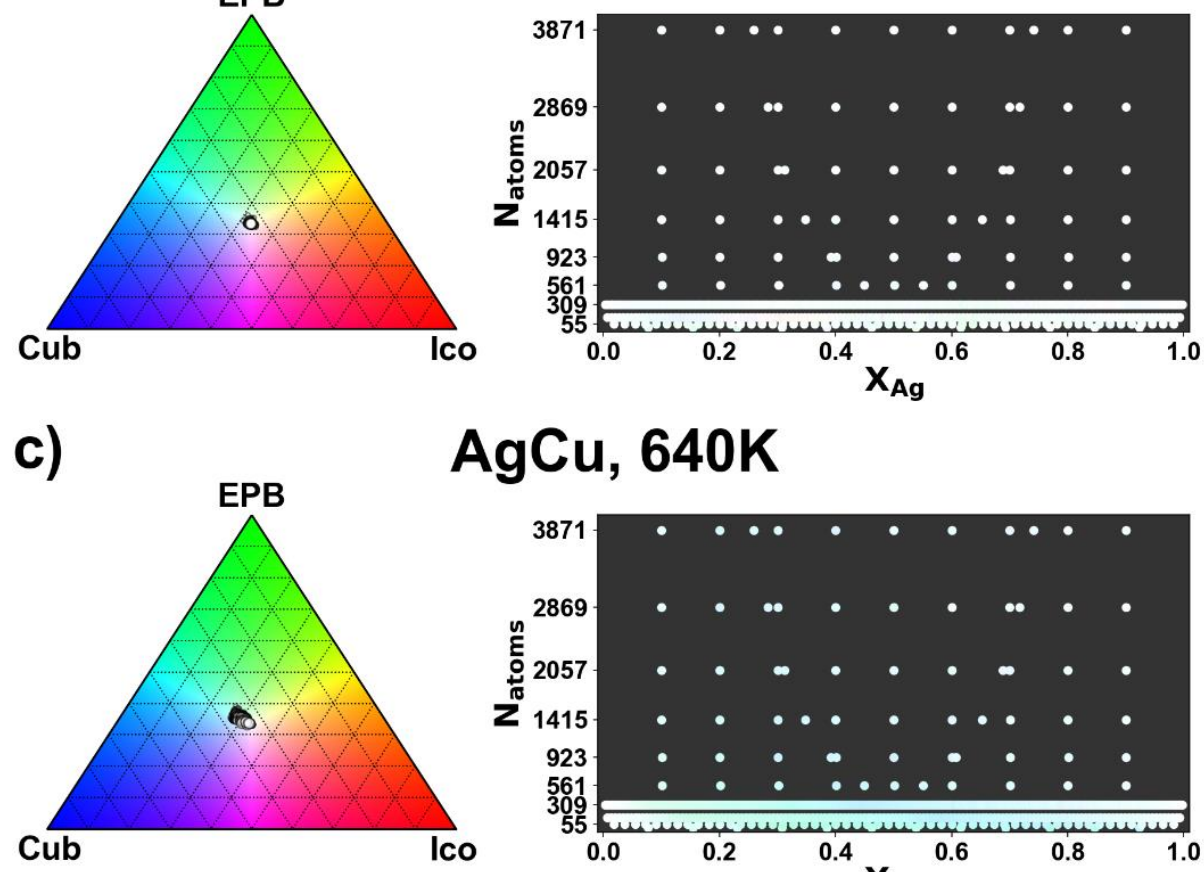

$\mathrm{AgCu}, 640 \mathrm{~K}$

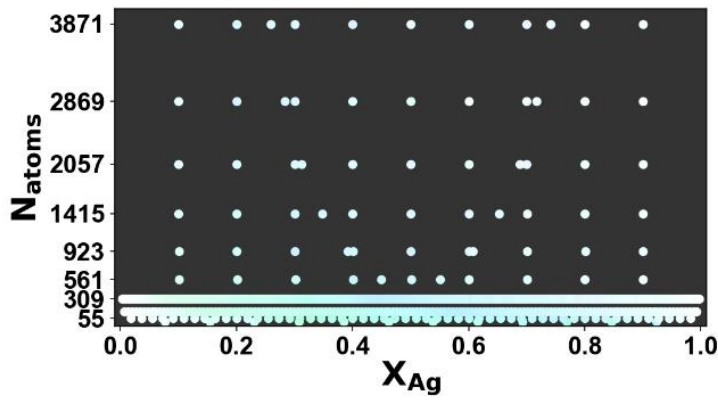

Figure S2. Morphology phase diagrams for the a) $\mathrm{AuCu}, \mathrm{b}) \mathrm{AgAu}$, and c) $\mathrm{AgCu}$ systems at $640 \mathrm{~K}$. The righthand column plots the preferred morphological phase as a function of the number of atoms and composition of the system. The legend is given by the ternary diagrams on the left-hand side, which show the percentage of the Boltzmann population taking on cuboctahedral, icosahedral, or EPB. White points, for example, indicate all three morphologies are equally favorable. In addition, the size of the points on the ternary diagrams corresponds to NP size. 


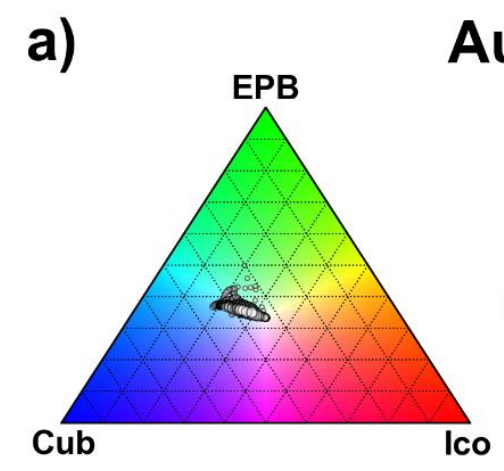

AuCu, 1073K
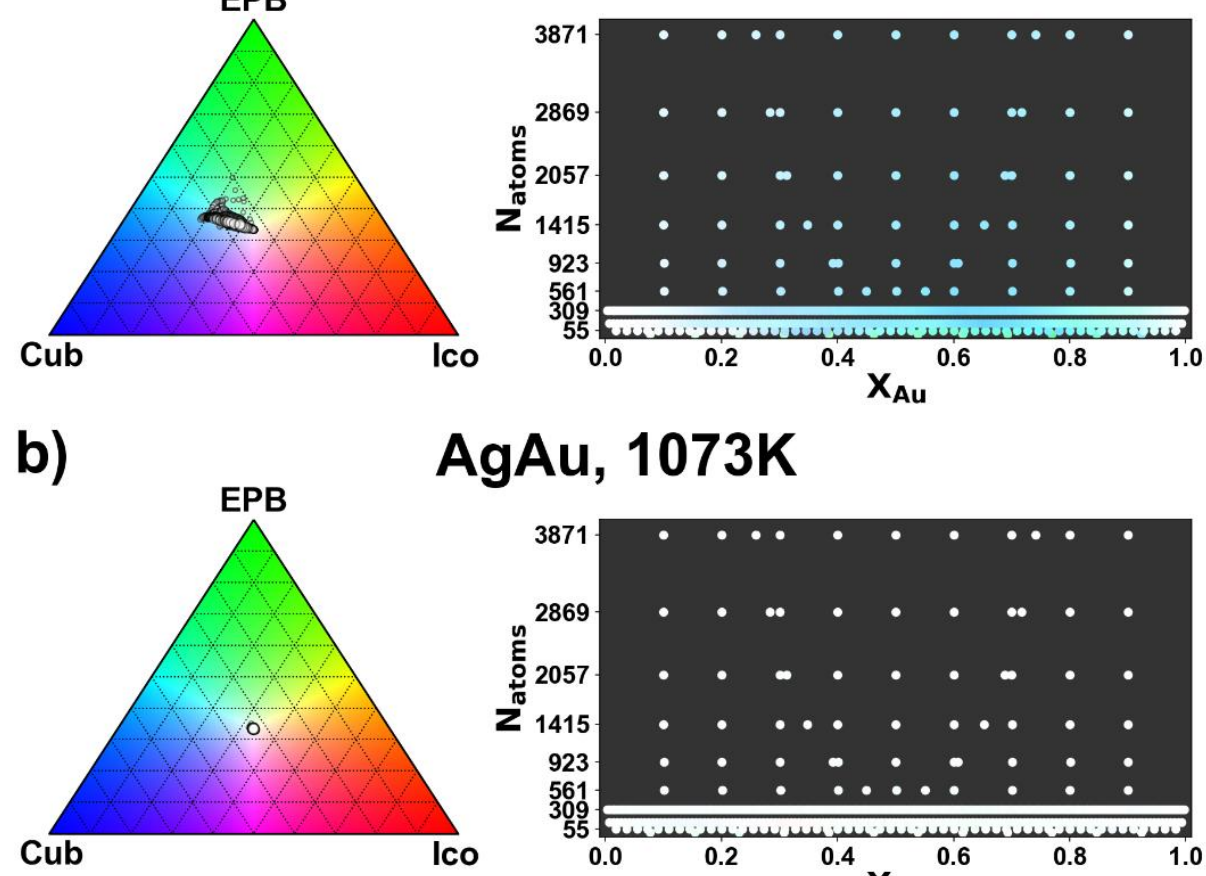

AgAu, 1073K
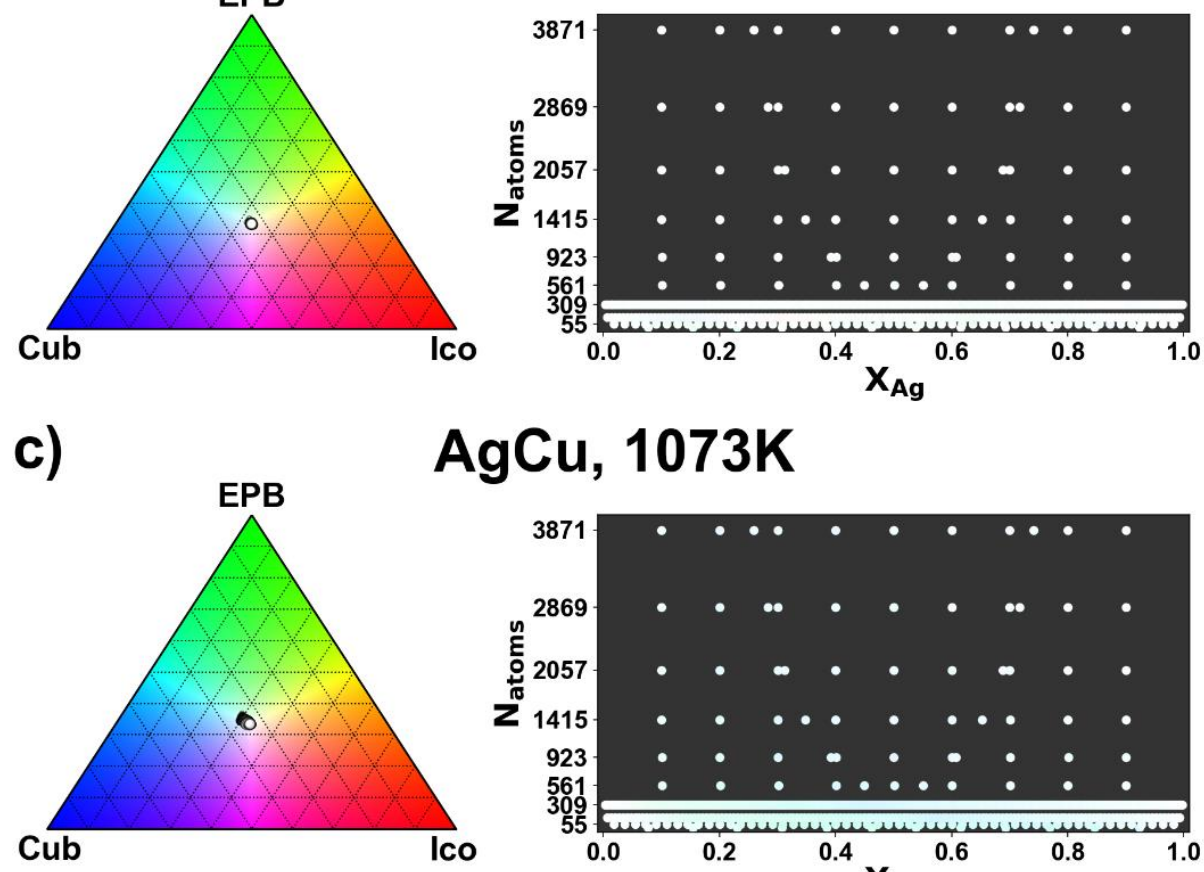

$\mathrm{AgCu}, 1073 \mathrm{~K}$

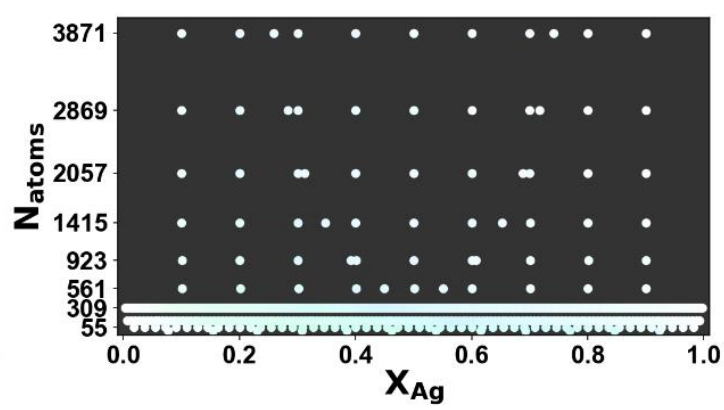

Figure S3. Morphology phase diagrams for the a) $\mathrm{AuCu}, \mathrm{b}) \mathrm{AgAu}$, and c) $\mathrm{AgCu}$ systems at $1073 \mathrm{~K}$. The righthand column plots the preferred morphological phase as a function of the number of atoms and composition of the system. The legend is given by the ternary diagrams on the left-hand side, which show the percentage of the Boltzmann population taking on cuboctahedral, icosahedral, or EPB morphology. White points, for example, indicate all three morphologies are equally favorable. In addition, the size of the points on the ternary diagrams corresponds to NP size.

\section{References}

1. Yan, Z.; Taylor, M. G.; Mascareno, A.; Mpourmpakis, G., Size-, Shape-, and CompositionDependent Model for Metal Nanoparticle Stability Prediction. Nano Lett. 2018, 18, 2696-2704.

2. Cramer, C. J., Essentials of Computational Chemistry: Theories and Models; J. Wiley: West Sussex, England ; New York, 2002, p 542.

3. Kittel, C., Introduction to Solid State Physics, 8th ed.; Wiley: Hoboken, NJ, 2005, p 680. 